\title{
Le risque accidentogène d'une consommation de stupéfiants est-il bien établi ?
}

\section{Is the risk of road crash after a drugs of abuse intake well documented?}

Patrick MURA*, Yves PAPET, Gérard MAUCO

Laboratoire de Biochimie et Toxicologie, Centre Hospitalier Universitaire - BP 577 - 86021 POITIERS - FRANCE

Tél : 0549443923 - Fax : 0549443834 - E-mail : p.mura@chu-poitiers.fr

* Auteur à qui adresser la correspondance : Dr Patrick MURA, Laboratoire de Biochimie et Toxicologie,

Centre Hospitalier Universitaire - BP 577 - 86021 POITIERS - FRANCE

Tél : 0549443923 - Fax : 0549443834 - E-mail :p.mura@chu-poitiers.fr

\section{$R \hat{E} S U M E ́$}

Une étude multicentrique cas-témoins, réalisée récemment en France, a montré que la fréquence des accidents corporels de la voie publique était multiplié par 2,5 (odds-ratio) avec un usage récent de cannabis et par 8,2 lorsque la morphine était présente dans le sang des conducteurs. Ces résultats confirment les données obtenues par de très nombreuses études de prévalence réalisées en France et dans de nombreux autres pays. Ce risque de survenue d'accidents induit par une consommation récente de stupéfiants n'est cependant pas mis en évidence uniquement par ces études épidémiologiques. Il existe en effet de nombreux autres arguments scientifiques. Le cannabis, les amphétamines, la cocaine et les opiacés sont des substances psychoactives, modifiant de manière significative la libération et/ou la recapture de certains neurotransmetteurs au niveau des synapses neuronales, ce qui se traduit par une altération des fonctions cognitives et motrices. Les conséquences de ces dysfonctionnements neuronaux (notamment les troubles de la vision, de la vigilance et de l'équilibre) peuvent être objectivées par des tests comportementaux. Par ailleurs, les études réalisées sur simulateurs de conduite ou sur circuits montrent que l'usage de ces stupéfiants augmente le risque d'erreurs de conduite. Prenant en compte l'ensemble de ces arguments, les auteurs en concluent que le risque accidentogène lié à une consommation de stupéfiants est aujourd'hui bien établi et qu'une large information auprès des conducteurs et en particulier auprès des jeunes est désormais nécessaire et urgente.

\section{MOTS-CLÉS}

Cannabis, amphétamines, cocaïne, opiacés, stupéfiants, conduite automobile, accidents.

\section{SUMMARY}

A collaborative French case-control study revealed that the number of non-fatal accidents was increased by 2.5 (oddsratio) with a recent intake of cannabis and by 8.2 when morphine was present in blood of drivers. These data confirm the results of numerous prevalence studies performed in France and many other countries. Moreover, the causal role for drugs of abuse in road crashes is not only attested by epidemiological studies, and numerous additional scientific arguments exist. Cannabis, amphetamines, cocaine and opiates are psychoactive compounds, modifying significantly the release and/or the recapture of neurotransmitters in synaptic areas, which leads to alterations of both motor and cognitive functions. The consequences of such neuronal dysfunctions (and particularly vision, vigilance and equilibrium troubles) can be highlighted by behavioral tests. Studies performed by driving simulators or even in "realistic" situations on closed or open -roads demonstrate that a recent intake of drugs of abuse increases the risk of driving faults. Taking into account all these arguments, the authors conclude that the risk of accident in case of drug of abuse exposu$r e$ is now well established and that it is necessary to address informations about this risk to drivers and more particularly young drivers.

\section{KEY-WORDS}

Cannabis, cocaine, amphetamines, opiates, drugs of abuse, car driving, road crashes. 


\section{Introduction}

Dans de très nombreux pays, des législations ont été mises en place visant à sanctionner les conducteurs sous influence de stupéfiants (1-4). Ces actions ont été consécutives à la prise de conscience des effets délétères de ces substances sur l'aptitude à conduire un véhicule en toute sécurité. En effet, depuis très longtemps, des rapports scientifiques alarmants ont été publiés, et portés à la connaissance de la collectivité scientifique et médicale internationale comme par exemple lors des Congrès de l'International Council on Alcohol, Drugs and Traffic Safety (5). En France, il a fallu attendre 2003 pour que soit créé un délit spécifique applicable à tout conducteur ayant fait usage de substances ou plantes classées comme stupéfiants $(6$, 7). Ce faisant, la France se soumettait donc à la Directive Européenne ( ${ }^{\circ}$ 91/439/CEE) du 29 juillet 1991, applicable au $1^{\text {er }}$ juillet 1996, qui imposait aux pays membres de l'Union Européenne le texte suivant : «le permis de conduire ne doit être ni délivré ni renouvelé à tout candidat ou conducteur en état de dépendance vis-à-vis de substances à action psychotrope ou qui, sans être dépendant, en abuse régulièrement.... ou qui consomme régulièrement des substances psychotropes, quelle qu'en soit la forme, susceptibles de compromettre son aptitude à conduire sans danger, si la quantité absorbée est telle qu'elle exerce une influence néfaste sur la conduite. Il en est de même pour tout autre médicament ou association de médicaments qui exerce une influence sur l'aptitude à conduire...».

Ce retard français semble être avoir été essentiellement dû au fait que certains conseillers estimaient que le risque accidentogène lié à une consommation de stupéfiants n'était pas encore clairement établi. Ainsi par exemple, le Professeur Claude Got précisait que «le surrisque d'accident lié à l'usage de stupéfiants ne fait pas l'objet d'un consensus pour deux raisons : les produits sont multiples et les études disponibles ne permettent pas de documenter avec facilité un surrisque dans les conditions de la circulation réelle, en se fondant sur des études accidentologiques et toxicologiques comparant un groupe d'accidentés et un groupe témoin représentatif du groupe exposé au risque» (8). Même s'il semble pour le moins surprenant que toutes les dispositions législatives en cours aux Etats-Unis et dans la plupart des pays européens aient été instituées sans fondement, nous avons toutefois entrepris dans cet article de rassembler les différents arguments mettant en évidence le lien entre consommation récente de stupéfiants et survenue d'accidents de la voie publique.

\section{Non pas un argument, mais un faisceau d'arguments}

La mise en évidence des risques d'accidents liés à un usage récent de stupéfiants repose sur différentes connaissances : les mécanismes d'action des principes actifs de ces substances et leurs effets sur le comportement des consommateurs, les données apportées par des études sur simulateur de conduite, les tests comportementaux, les tests en situation réelle, ainsi que les résultats d'études épidémiologiques.

Étudier ce problème sous le seul angle des études épidémiologiques est donc tout à fait réducteur.

\section{Mécanismes d'action et effets induits chez les conducteurs (9)}

Les récepteurs et leurs localisations au niveau du cerveau sont désormais bien connus (10). Les mécanismes impliqués dans la voie hédonique, concourant au sentiment de plaisir procuré par ces produits, sont aujourd'hui mieux documentés, aboutissant notamment en une augmentation de la libération de dopamine au niveau du noyau accumbens et de l'aire tegmentale ventrale. Il en est de même pour les mécanismes responsables des perturbations cognitives et motrices.

L'ecstasy stimule la production de sérotonine et inhibe sa recapture. Cela se traduit par des effets stimulants et euphorisants, ayant comme conséquences principales un comportement irrationnel et des prises de risques accrues.

La cocaïne inhibe la recapture de la dopamine au niveau du système limbique, ce qui procure au consommateur un sentiment de très grande confiance en soi et l'amène à prendre des risques inconsidérés:

Les opiacés, par leurs actions au niveau des récepteurs $\mu$, $\kappa$ et $\lambda$, provoquent une sédation pouvant être majeure avec diminution des réflexes et de l'attention associée à des troubles visuels.

Le principe actif du cannabis, en augmentant la production de dopamine principalement au niveau de l'hippocampe, du cortex frontal, du cervelet et des ganglions de la base, est responsable de nombreux effets délétères sur la conduite : diminution de la vigilance, diminution des réflexes, mauvaise estimation des distances, vision nocturne altérée, etc.

Quelque soit le stupéfiant concerné parmi les produits sus-cités, il est important de noter qu'en cas de consommation régulière et importante, $d$ 'autres effets néfastes apparaissent. Par exemple, lors d'un usage fréquent et prolongé de cannabis, des crises d'angoisse aiguë peuvent survenir au cours desquelles un véritable état de panique s'installe. Par ailleurs, un syndrome 
amotivationnel est classiquement observé chez les usagers chroniques (11). Il y a donc chez ces sujets un état permanent d'incapacité à conduire un véhicule en toute sécurité.

\section{Études sur simulateurs de conduite}

De très nombreuses études sur simulateurs de conduite ont montré des altérations significatives de la capacité à conduire un véhicule observables chez les sujets ayant consommé des stupéfiants.

Barnett et coll (12) ont montré, chez des sujets ayant fumé une cigarette de cannabis dosée à 100, 200 ou 250 $\mu \mathrm{g} / \mathrm{kg}$ de poids corporel, que les effets négatifs (augmentation du temps de réponse, sorties de route) du cannabis sur les performances de conduite étaient à leur maximum 15 min après consommation et qu'ils étaient observables pendant plusieurs heures (2 à 7 selon les paramètres étudiés). Par ailleurs ces auteurs ont montré, chez des sujets ayant fumé des cigarettes contenant différentes concentrations en principe actif, l'existence d'une relation significative entre le nombre d'erreurs de conduite et la concentration en principe actif dans le sang.

Liguori et coll., chez des sujets ayant consommé des cigarettes dosées à $3,75 \%$ de THC, ont montré que le temps de réaction au freinage était augmenté de $55 \mathrm{ims}$ $(\mathrm{p}<0,1)$, ce qui correspond à une distance de freinage $\mathrm{de}+1,5 \mathrm{~m}$ à $90 \mathrm{~km} / \mathrm{h}(13)$.

\section{Les résultats de tests comportementaux}

Selon la plupart des études, l'exposition au cannabis entraîne une altération des performances psychomotrices lors de l'accomplissement de tâches complexes liée aux troubles de l'attention, de la coordination perceptivomotrice et à l'allongement du temps de réaction $(14,15)$. Une diminution de vitesse de la poursuite visuelle dans le champ central et périphérique est observée pendant plus de 5 heures après 15 minutes d'inhalation de cannabis (16). Les perturbations psychomotrices induites par une consommation de cannabis limitent ainsi les activités que les individus sont susceptibles de faire de manière efficace ou en toute sécurité comme la conduite automobile (11).

De nombreux travaux ont montré que l'usage d'amphétamines ou de cocaïne altérait de façon tout à fait significative les performances cognitives et psychomotrices (17-19).

\section{Les tests en situation réelle}

Les études effectuées sur route confirment les résultats observés sur simulateurs de conduite. Le cannabis affecte le contrôle de trajectoire en augmentant de manière significative et dose-dépendante la variabilité du contrôle latéral (écart à la bordure de la voie) (20) et augmente la distance de freinage (13).

De tels tests sont irréalisables en France pour des raisons d'ordre éthique et légal. Une expérience française a été effectuée en 1998 par des journalistes, sous contrôle de scientifiques et de médecins anonymes (21). Leur étude a porté sur différents types de consommateurs : très occasionnels (quelques prises par an), occasionnels (quelques prises par mois) et réguliers (plusieurs prises par semaine). Après avoir consommé du cannabis, leurs aptitudes à conduire un véhicule ont été évaluées sur circuit fermé. Les principaux résultats ont été les suivants :

- des perturbations très notables de la vision, surtout de nuit : temps de récupération après éblouissement augmenté, mauvaise appréciation des distances, erreurs de vision des couleurs,

- des sorties de trajectoire en virage,

- des temps de réaction augmentés avec des distances de freinage très allongées (plus 5 à $12 \mathrm{~m}$ à $80 \mathrm{~km} / \mathrm{h}$ ),

- des difficultés à réaliser des marches arrière en tournant.

\section{Les études épidémiologiques}

Les études réalisées en France ces dernières années (22-26) ont montré que certaines substances psychoactives illicites étaient fréquemment retrouvées dans les urines et/ou le sang de conducteurs impliqués dans un accident de la voie publique. Toutes ces études ont révélé que le cannabis était la drogue illicite la plus fréquemment détectée. A caractère purement descriptif, ces études ont permis de révéler l'ampleur du phénomène. En effet, l'absence de population témoin et/ou l'utilisation de l'urine comme milieu biologique d'investigation ne leur avaient pas permis d'établir un lien de causalité entre cette consommation et la survenue de l'accident.

Une récente étude multicentrique (27) a permis de confirmer et de quantifier le risque relatif d'accident associé à un usage récent de substances psychoactives. Les auteurs ont analysé le sang de 900 conducteurs impliqués dans un accident corporel et comparé ces résultats à ceux de 900 sujets témoins. Les analyses, réalisées sur le sang à l'aide des méthodes analytiques les plus performantes à ce jour, concernaient les produits suivants : cannabis, amphétamines, opiacés, cocaïne, alcool, ainsi que la recherche des principaux médicaments psychoactifs.

Des différences de prévalences très significatives $(\mathrm{p}<0,01)$ étaient observées chez les moins de 27 ans pour le cannabis (le THC était seul présent chez 15,3\% des conducteurs et $6,7 \%$ des témoins), quel que soit l'âge pour morphine $(2,7 \%$ des conducteurs et $0,3 \%$ 
des témoins) et pour l'alcool (17\% des conducteurs et $5 \%$ des témoins). Parmi les conducteurs positifs au cannabis, celui-ci était seul présent chez $60 \%$ d'entre eux, alors que le discours officiel tente de faire croire à un usage systématique avec l'alcool éthylique.

L'analyse statistique de ces résultats (calcul des oddsratios) a permis aux auteurs de montrer que, chez les moins de 27 ans, la fréquence des accidents était multipliée par (entre parenthèses, l'intervalle de confiance à $95 \%)$ :

$>1,7(1,2-2,4)$ avec les benzodiazépines,

$>2,5(1,5-4,2)$ avec le cannabis seul,

$>3,8(2,1-6,8)$ avec l'alcool seul,

$>4,6(2,0-10,7)$ avec l'association alcool-cannabis

$>$ et par 8,2 (2,5-27,3) avec la morphine.

Ces résultats ont ainsi clairement démontré qu'une consommation récente de substances psychoactives était de nature à fortement altérer les capacités à conduire un véhicule en toute sécurité, et tout particulièrement chez les jeunes conducteurs.

Une étude réalisée en Guadeloupe (28) sur 94 conducteurs et 94 témoins de moins de 30 ans et utilisant un protocole similaire a révélé un odds-ratio de $2,7(1,31$ $5,61)$ pour le cannabis, confirmant ainsi les données obtenues en France métropolitaine (27).

\section{Discussion et conclusion}

Les études de prévalence sont intéressantes car elles permettent de quantifier l'ampleur du phénomène dans un pays donné et à un moment donné. Ainsi de nombreuses études $(22,24-26,29)$ ont montré qu'en France, l'alcool n'était plus le seul produit psychoactif retrouvé dans l'organisme des conducteurs impliqués dans des accidents de la voie publique.

Les études épidémiologiques visant à quantifier le risque accidentogène de telle ou telle substance sont beaucoup plus difficiles à mettre en œuvre. Elles nécessitent la présence d'une population témoin, un large échantillonnage, mais aussi l'utilisation de méthodes analytiques spécifiques et sensibles. Afin de pouvoir rechercher tous les facteurs de confusion possibles, le protocole analytique «idéal» consisterait à entreprendre en parallèle plusieurs méthodologies : chromatographie gazeuse couplée à la spectrométrie de masse (cannabis, amphétamines, cocaïne, opiacés, méthadone, etc.), chromatographie liquide haute performance avec détection à barrettes de diodes (principaux médicaments psychoactifs, caféine, etc.), chromatographie liquide couplée à la spectrométrie de masse (LSD, buprénorphine, flunitrazépam, etc.). Ces analyses doi- vent de plus être réalisées sur le sang, seul milieu biologique permettant de mettre en évidence une consommation récente (30). Par ailleurs, les connaissances sur la toxicocinétique sanguine des cannabinoïdes imposent que les prélèvements sanguins soient effectués dans l'heure qui suit le moment de l'accident (31).

Ainsi, dans une étude cas-témoins réalisée en Australie, Longo et coll. (32) avaient conclu à l'absence de lien de causalité entre la prise de cannabis ou d'amphétamines et la survenue d'accident. Cependant, le seuil de positivité utilisé dans cette étude pour le THC dans le sang était de $40 \mathrm{ng} / \mathrm{ml}$ : une telle concentration ne pouvant être observée que dans les minutes qui suivent la consommation (31), aucun crédit ne peut être apporté aux conclusions de cette étude. Les effets du cannabis étant encore présents lorsque les concentrations en principes actifs (THC et 11-OH THC) sont devenues inférieures à $1 \mathrm{ng} / \mathrm{ml}$, il faut considérer que la seule présence de principe actif dans le sang permet d'affirmer que le sujet a récemment consommé et donc d'estimer qu'il était sous influence de cannabis au moment du prélèvement. Kudo et coll. (33) ont montré, en analysant les tissus d'un sujet décédé accidentellement, des concentrations en THC dans le sang et dans le cerveau respectivement de 1,85 et $9,78 \mathrm{ng} / \mathrm{g}$. Si la notion de seuil sanguin de dangerosité pour le cannabis est donc un nonsens au plan scientifique, il est bien entendu indispensable d'établir un seuil de positivité. Compte tenu des performances techniques actuelles (sensibilité, spécificité, reproductibilité), le seuil proposé par la Société Française de Toxicologie Analytique est de $0,5 \mathrm{ng} / \mathrm{ml}$ pour chacun des cannabinoïdes.

Lors d'une étude française réalisée en 1992 sur 2938 sujets hospitalisés en urgence après un accident de la voie publique (34), 6,6\% des conducteurs étaient positifs au cannabis. Ce résultat doit être considéré avec la plus grande réserve, puisque les analyses avaient été réalisées sur du plasma par méthode immunoenzymatique, non adaptée à ce milieu biologique, insuffisamment sensible et spécifique.

A notre connaissance, l'étude multicentrique française (27) est la seule étude ayant été réalisée avec un protocole se rapprochant le plus possible du protocole «idéal». Cette étude a très nettement conclu à l'existence d'un lien de causalité significatif entre la consommation récente de cannabis ou de morphiniques et le risque d'accident.

Les résultats d'une vaste étude épidémiologique instituée en France par voie réglementaire devraient être publiés en 2004. Cette étude, se déroulant sur la période d'octobre 2001 à octobre 2003, est réalisée sur tous les conducteurs impliqués dans un accident immédiatement mortel, en utilisant les résultats d'analyses san- 
guines obtenues par 40 laboratoires agréés pour ce faire. Une telle étude devrait permettre de mieux quantifier le risque d'accident lié à la consommation de stupéfiants, à condition qu'il soit établi que les prélèvements sanguins auront été effectués dans des délais d'une heure au plus après les accidents, que les 40 laboratoires impliqués dans cette étude disposaient du matériel nécessaire et avaient obtenu de bon résultats à un contrôle de qualité externe concernant le dosage des stupéfiants dans le sang.

En raison de toutes ces difficultés de mise en place et d'interprétation d'études épidémiologiques, tous les autres arguments doivent donc être pris en compte.

Pour affirmer que telle ou telle substance est susceptible d'altérer les capacités de conduire un véhicule en toute sécurité, il pourrait en effet suffir de démontrer que l'usage de cette substance modifie de manière péjorative les fonctions cognitives et motrices des individus. C'est logiquement le cas pour toute substance ayant des propriétés psychoactives, parmi lesquelles figurent le cannabis, les amphétamines, la cocaïne et les opiacés. Il apparaît en effet difficile de concevoir que ces produits, qui altèrent de façon significative la vigilance, l'équilibre, les réflexes et la vision, ne perturbent pas l'aptitude à réaliser en toute sécurité un acte aussi complexe qu'est la conduite automobile. Les tests comportementaux, les résultats des études effectuées sur simulateurs de conduite ou sur circuit ne font que confirmer cet état de fait.

En conclusion et à la lumière de tous ces éléments, conduire sous l'influence de stupéfiants accroît le risque de survenue d'accidents de la voie publique. A une époque où la consommation de cannabis et d'ecstasy ne cesse d'augmenter dans la plupart des pays et en particulier en France (35), il convient donc aujourd'hui de procéder auprès des jeunes à une large information de ces risques.

\section{Références}

1. Charlier C., Verstraete A., Plomteux G. La législation dans les pays européens. In : Mura P., ed. Alcool, médicaments, stupéfiants et conduite automobile. Paris : Elsevier, 1999 ; 215-32.

2. Verstraete A. Stupéfiants et conduite automobile - Les actions réalisées en Belgique. Ann. Toxicol. Anal. 2003 ; $15: 128-137$.

3. Augsburger M. Stupéfiants et conduite automobile - Les actions réalisées en Suisse. Ann. Toxicol. Anal. 2003 ; $15: 138-144$

4. Moeller M.R. Drugs of abuse and driving-legal conditions and law enforcement in Germany. Ann. Toxicol. Anal. $2003 ; 15: 145-152$.
5. De Gier J.J. Fifty years of research into the pharmacology and toxicology of drugs other than alcohol with reference to traffic safety. In : T 2000, Alcohol, Drugs and Traffic Safety. Stockholm : Ekonomi-Print, 2000 : 9-18.

6. Journal Officiel de la République Française, 4 février 2003, p. 2103.

7. Journal Officiel de la République Française, ler avril 2003, p. 5702.

8. http://www. securite-routiere.org

9. Anger J.P. Effets des stupéfiants sur la conduite automobile. Ann: Toxicol. Anal. $2003 ; 15: 71-76$

10. Julien R.M. In : A primer of drug action. New York, W.H. Freeman and Company, 1997.

11. Expertise collective INSERM. Cannabis. Quels effets sur le comportement et la santé ? In : INSERM, ed. Paris : Jouve, 2001.

12. Barnett G., Licko V., Hompson T. Behavioral pharmacokinetics of marijuana. Psychopharmacol. 1985 ; 85 : 51-6.

13. Liguori A., Gatto C.P., Robinson J.H. Effects of marijuana on equilibrium, psychomotor performance, and simulated driving. Behav. Pharmacol. 1998 ; 9 : 599-609.

14. Hall W., Solowij N., Lemon L. The health and psychological consequences of cannabis use. Monograph series. Department of human services and health eds, 1994.

15. Ameri A. The effects of cannabinoids on the brain. Prog. Neurobiol. 1999 ; 58 : 315-48.

16. Fant R.V., Heishman S.J., Bunker E.B., Pickxorth W.B. Acute and residual effects of marijuana in humans. Pharmacol. Biochem. Behav. 1998 ; 60 : 777-84.

17. Simon S.L., Domier C.P., Sim T., Richardson K., Rawson R.A., Ling W. Cognitive performance of current methamphetamine and cocaine abusers. J. Addict. Dis. 2002 ; $21: 61-74$.

18. Fillmore M.T., Rush C.R., Hays L. Acute effects of oral cocaine on inhibitory control of behavior in humans. Drug. Alcohol Depend. 2002 ; 67 : 157-67.

19.Zancaner S., Giorgetti R., Dal Pozzo C., Molinari G., Snenghi R., Ferrara S.D. Driving under the influence of drugs. Correlation between clinical signs and type of intoxication. In: Mercier-Guyon eds., Alcohol, Drugs and traffic Safety. Annecy, 1997 : 757-68.

20. Robbe HWJ. Marijuana's impairing effects on driving are moderate when taken alone but severe when combined with alcohol. Human Psychopharmacol. Clin. Exp. $1998 ; 13$ : S70-S78.

21. Auto Plus. Drogue au volant, Auto Plus a testé. Magazine Auto Plus 1998 ; 505 : 48-53.

22. Deveaux M., Marson J.C., Goldstein P., Lhermitte M., Muller P.H. Alcool et médicaments psychotropes dans les accidents mortels de la circulation. Etude de 132 cas dans la région Nord-Pas-de-Calais. Sem. Hôp. Paris 1991 : 1372-6.

23. Marquet P., Delpla P.A., Kerguelen S. Prevalence of drugs of abuse in urine of drivers involved in road accidents in France : a collaborative study. J. Forensic Sci. $1998 ; 43: 806-11$. 
24.Pépin G., Mura P., Kintz P., Dumestre V., Ghysel M.H., Goulle J.P. Recherche de stupéfiants dans le sang de conducteurs d'automobiles : résultats d'une compilation française d'expertises toxicologiques. Toxicorama 1999; $11: 12-6$.

25. Mura P., Pépin G., Marquet P., Goullé J.P., Deveaux M., Tourneau J., Kintz P. Place des stupéfiants dans les accidents mortels et corporels de la voie publique. Résultats de 169 analyses sanguines réalisées en 1998 et 1999 à la demande d'une autorité judiciaire. Toxicorama 1999 ; $11: 225-31$

26. Kintz P., Cirimele V., Mairot F., Muhlmann M., Ludes B. Analyses toxicologiques pratiquées sur 198 conducteurs accidentés. Presse Med. 2000 ; 29 : 1275-8.

27. Mura P., Kintz P., Ludes B., Gaulier J.M., Marquet P., Martin-Dupont S., Vincent F., Kaddour A., Goullé J.P., Nouveau J., Moulsma M., Tilhet-Coartet S., Pourrat O. Comparison of the prevalence of alcohol, cannabis and other drugs between 900 injured drivers and 900 control subjects : results of a French collaborative study. Forensic Sci. Int. $2003 ; 133$ : 79-85.

28. Ragoucy-Sengler C., Wind H., Simonetti M., Daijardin J.B., Kintz P., Pileire B. Conduite sous l'influence de stupéfiants et de psychotropes en Guadeloupe. Ann. Toxicol. Anal. 2003 ; 15 (sous presse).

29. Marc B., Bontemps V., Baudry F., Richard B., Ghaith A., Garnier M. Drugs of abuse in urine of young adult drivers involved in road accidents in a Paris suburb. J. Clin. Forensic Med. $2000 ; 7: 77-81$.

30. Kintz P., Samyn N., Verstraete A. Les prélèvements biologiques et techniques analytiques. In : Mura P., ed. Alcool, médicaments, stupéfiants et conduite automobile. Paris : Elsevier, 1999 ; 143-169.

31. Mura P., Brunet B., Monzon C., Papet Y., Mauco G. Interprétation des concentrations sanguines en cannabinoïdes : données actuelles. Ann. Toxicol. Anal. 2003 ; $15: 35-9$.

32. Longo M.C., Hunter C.E., Lokan R.J., White J.M., White M.A. The prevalence of alcohol, cannabinoids, benzodiazepines and stimulants amongst injured drivers and their role in driver culpability - Part II : The relationship between drug prevalence and drug concentration, and driver culpability, Accident Analysis and Prevention 2000 ; 32 : 623-32.

33. Kudo K., Nagata T., Kimura K., Imamura T., Jitsufuchi N. J. Anal. Toxicol. $1995 ; 19: 87-90$.

34. Schermann J.M. Evaluation du risque d'accident de la circulation lié à l'absorption de drogues illicites. In : Convention Direction de la Sécurité Routière, Rapport final, 1992.

35. ESCAPAD 2001. Santé, mode de vie et usages de drogues à 18 ans. OFDT, Paris, juin 2002. 